\title{
Descrição da larva, exúvia pupal e macho de Leucotabanus albovarius (Walker) (Diptera, Tabanidae) da Amazônia Central
}

\author{
Fabio Siqueira Pitaluga de Godoi ${ }^{1,3} \&$ José Albertino Rafael ${ }^{2,3}$
}

${ }^{1}$ Bolsista do CNPq, Programa de pós-graduação em Entomologia. fgodoi@gmail.com
${ }^{2}$ Bolsista de produtividade do CNPq. jarafael@inpa.gov.br
${ }^{3}$ Coordenação de Pesquisas em Entomologia, Instituto Nacional de Pesquisas da Amazônia, Caixa Postal 478, 69011-970, Manaus-AM, Brasil.

\begin{abstract}
Description of larva, pupal exuvia and male of Leucotabanus albovarius (Walker) (Diptera, Tabanidae) from Central Amazonia. Immature stages and male adult of Leucotabanus albovarius (Walker, 1854) are described and figured for the first time. The larva was found in rotten wood of the palm Bactris gasipaes Kunth (Arecaceae) near Manaus. Differential diagnoses of the known immatures of Leucotabanus Lutz are presented.
\end{abstract}

KEYWORDS. Brachycera; Brazil; horsefly; taxonomy.

RESUMO. Descrição da larva, exúvia pupal e macho de Leucotabanus albovarius (Walker) (Diptera, Tabanidae) da Amazônia Central. Estágios imaturos e macho adulto de Leucotabanus albovarius (Walker, 1854) são descritos e ilustrados pela primeira vez. A larva foi encontrada em tronco de pupunheira em decomposição, Bactris gasipaes Kunth (Arecaceae), nas proximidades de Manaus. Diagnoses diferenciais dos imaturos de Leucotabanus Lutz são apresentadas.

PALAVRAS CHAVE. Brachycera; Brasil; mutuca; taxonomia.

Os tabanídeos são relativamente bem conhecidos na fase adulta por meio das fêmeas, mas o conhecimento das fases imaturas e dos machos é incipiente. Das quase 1.200 espécies neotropicais (Fairchild \& Burguer 1994) apenas 50 são conhecidas de formas imaturas (Coscarón et al. 1996), sendo grande parte espécies panamenhas descritas por Goodwin \& Murdoch (1974). Um número um pouco maior de espécies apresentam machos conhecidos. O conhecimento dos imaturos e dos machos pode ajudar a resolver problemas taxonômicos. Leucotabanus Lutz, 1913 contém 17 espécies, sendo 15 neotropicais e duas neárticas (Fairchild 1985). As espécies com formas imaturas e machos conhecidos estão indicadas na Tabela I que apresenta os sexos e as formas jovens conhecidas das 17 espécies. As larvas das espécies conhecidas foram coletadas em madeira em decomposição, em troncos mortos ou em buracos de árvores vivas (Fairchild 1985, 1986). $L$. albovarius (Walker, 1854) tem ocorrência na floresta amazônica da Guiana, Guiana Francesa, Suriname, Peru, Bolívia e Norte do Brasil (Amazonas, Pará e Rondônia) (Fairchild \& Burger 1994), sendo a quinta espécie a ser descrita de formas imaturas e a oitava a ter o macho conhecido no gênero.

\section{MATERIALE MÉTODOS}

Duas larvas de Leucotabanus albovarius (Walker) foram coletadas em tronco de pupunheira em decomposição, Bactris gasipaes Kunth (Arecaceae), no Sítio Vida Tropical (0251'47"S - 59 $\left.{ }^{\circ} 55^{\prime} 55^{\prime \prime} \mathrm{W}\right)$, localizado próximo a cidade de Manaus, no Ramal Água Branca II da Rodovia AM-010, km-35.

Após a emergência do adulto, a segunda larva que ainda não havia empupado foi fixada com KAAD e conservada em álcool 70\%; a exúvia pupal foi conservada em álcool 70\% e o macho adulto alfinetado.

Foram ainda coletados mais nove espécimes machos nos municípios de Manaus e Presidente Figueiredo por meio de coletas noturnas em lençol, utilizando luz mista de vapor de mercúrio e filamento de tungstênio e luz negra (BLB). Os espécimes coletados foram dissecados e sua genitália comparada com o espécime criado em laboratório.

O material está depositado na Coleção de Invertebrados do Instituto Nacional de Pesquisas da Amazônia - INPA, Manaus, Brasil.

A terminologia adotada segue McAlpine (1981) para a descrição dos caracteres morfológicos externos do adulto, Sinclair et al. (1994) para caracteres da terminália masculina e Goodwin \& Murdoch (1974) para a descrição dos estágios imaturos.

Informações adicionais estão entre colchetes ([]), completando dados omitidos nas etiquetas.

\section{Leucotabanus albovarius (Walker, 1854)}

(Figs. 1 - 18)

Adulto macho. Comprimento $17 \mathrm{~mm}$ (excluindo antenas). Cabeça mais larga que o tórax. Olho glabro, holóptico. Facetas dorsais castanhas, maiores, ocupando a maior parte do olho, distintamente delimitadas das facetas ventrais e marginais, menores, pretas (Fig. 1). Olho em vida com facetas maiores brônzeas e as menores verdes iridescentes (Figs. 14-15). Tubérculo ocelar pequeno, distinto, com três vestígios de ocelos distintos. Triângulo frontal com pruinosidade cinza, exceto o vértice superior castanho-amarelado em vista frontal. 
Antena (Fig. 2) com escapo e pedicelo castanho-claros, flagelo enegrecido com placa basal alaranjada. Palpo porrecto, amareloclaro. Mesonoto e escutelo com pilosidade branca e preta mesclada e pruinosidade cinza. Asa com basicosta setulosa, cerdas levemente mais esparsas que a área adjacente da veia costal. Pterostigma estreito, amarelo-claro. Abdômen. Tergitos I e II com pruinosidade e pilosidade branca, exceto base do tergito II com uma mancha de pêlos pretos lateralmente; tergitos III e IV castanhos com pruína castanha, tergito III com pêlos castanhos e com mancha triangular central de pêlos brancos na margem posterior e tergito IV com pêlos pretos na metade anterior e brancos na metade posterior; demais tergitos castanhos com pêlos pretos e pruinosidade cinza lateralmente, visível de acordo com o ângulo de incidência da luz. Esternitos castanho-claros com pêlos pretos exceto margem posterior com faixa estreita de pêlos brancos. Outros aspectos da fêmea, caracterizados por Fairchild (1985). Terminália. Epândrio longo e largo; margem anterior com uma forte escavação que se estreita na porção central e atinge a margem posterior dividindo a peça em duas partes; $1 / 2$ anterior estreita e curvada lateralmente; margem posterior projetada distalmente, com a porção apical marcada pela uma fissura longitudinal. Cercos unisegmentados, longos e largos, ultrapassando a margem distal do hipoprocto; margem proximal relativamente reta, enquanto a margem distal é levemente arredondada com uma leve reentrância na porção média. Hipoprocto quase totalmente encoberto pelos cercos em vista dorsal, com ápice estreito e arredondado e porção anterior contígua com o esclerito subepandrial. Esclerito subepandrial encoberto pelo epândrio, com exceção da porção mais anterior; aparentemente dividido em duas partes, mas com uma estrutura membranosa unindo ambas; porção anterior estreita com ápice afilado, porção posterior unida ao hipoprocto (Fig. 3). Hipândrio + gonocoxitos (Fig. 4) grande, mais longo do que largo e em forma de "ferradura"; margem anterior levemente arredondada. Margem posterior do hipândrio fortemente escavada anteriormente. Gonocoxitos longos e tubulares com as margens laterais paralelas, portando apódemas gonocoxais bem desenvolvidos, estreitos e subparalelos, ultrapassando a margem anterior do hipândrio + gonocoxitos. Gonóstilos longos, estreitos e convergentes, com uma acentuada depressão ao longo de toda extensão de sua face dorsal; margem apical média levemente deprimida projetando as margens laterais. Bainha parameral subtriangular e longa, com o ápice quase atingindo a margem posterior dos gonóstilos; margem anterior levemente escavada e ligada lateralmente aos gonocoxitos. Edeago (Fig. 5) simples, longo e em forma de "lança"; apódema ejaculatório longo e estreito, com porção anterior levemente alargada. Saco espermático de parede membranosa e transparente se estendendo desde a porção distal do apódema ejaculatório até final do guia edeagal. Processos ejaculatórios laterais pequenos, localizados na parede anterodorsal do saco espermático. Processo endoedeagal relativamente curto e largo, projetado posteriormente dentro do saco espermático. Guia edeagal estreito e alongado, com a porção anterior unida medialmente projetando as laterais anteriormente, onde sofrem uma forte curvatura direcionando os filamentos posteriormente, ultrapassando o processo endoedeagal até as duas partes se unirem formando uma espécie de ponta.

Variações. O espécime criado em laboratório apresentou algumas anomalias provavelmente em decorrência de uma alimentação deficiente no período em que foi mantido em laboratório e/ou da rápida fixação após a emergência.

Epândrio, cercos, hipoprocto e esclerito subepandrial perdidos durante a extração da genitália. Hipândrio + gonocoxitos (Fig. 6) com margem anterior arredondada. Hipândrio não evidenciando forte escavação anterior na margem posterior. Gonocoxitos divergentes. Gonóstilos com uma depressão apenas em uma pequena porção da face dorsolateral. Apódemas gonocoxais não observados provavelmente por estes terem sido perdidos durante a retirada da genitália ou durante a dissecção não sendo desta forma caracterizada como uma variação ou mesmo uma anomalia. Bainha parameral alongada e estreita, com o ápice ultrapassando a margem posterior dos gonóstilos. Edeago (Fig. 7) com saco espermático e guia edeagal retraídos formando um tipo de bainha na porção posterior, deixando o edeago curto e espessado.

Larva (Fig. 8): Comprimento do corpo $42 \mathrm{~mm}$. Larva brancocreme, exceto cápsula cefálica marrom (Fig. 16). Corpo alongado, delgado, desprovido de pilosidade conspícua. Cápsula cefálica com 1,67 mm de comprimento e 1,0 mm de largura, bem desenvolvida, esclerotinizada, com dois pares de cerdas subiguais dorsolaterais e três pares de cerdas ventrolaterais, o par anterior com cerdas aproximadas entre si e menor que as demais. Protórax mais longo e delgado que o meso e metatórax, com um par de cerdas ventrolaterais; espiráculo bilobado, dorsolateral na porção posterior (Fig. 9). Mesotórax ligeiramente menor que metatórax, ambos com um par de cerdas ventrolaterais cada. Abdômen com dois pares de pseudopódios no $1 / 4$ anterior dos segmentos I-VII, um ventral e um ventrolateral. Segmento VIII (anal) aproximadamente do mesmo comprimento que os segmentos VI e VII juntos, cilíndrico com porção posterior acuminada e dois pares de cerdas dorsolaterais e dois de ventrolaterais, o par posterior com as cerdas aproximadas entre si; espiráculo abdominal (segmento VIII) arredondado, conspícuo, posicionado dorsolateralmente no $1 / 4$ anterior do segmento (Fig. 10). Lobo anal bilobado.

Pupa. Castanha em vida (Figs. 17-18). Pupário castanhoclaro, com 38,3 mm de comprimento (Fig. 11). Tubérculo caloso, bainha antenal, saliência antenal, tubérculo frontal, sutura frontal e espiráculo torácico com esclerotinização mais acentuada. Placa frontal com 5,1 mm de comprimento e 3,9 mm de largura (Fig. 12). Tubérculo caloso subtriangular, rugoso, com uma cerda apical. Bainha antenal com 0,67 mm de comprimento e $0,58 \mathrm{~mm}$ largura, subtriangular, com segmentação distinta, seu ápice não atinge a sutura epicranial. Tubérculo frontal pequeno, pouco desenvolvido, levemente proeminente em vista lateral. Saliência antenal subtriangular em vista lateral, rugosa, apresentando duas protuberâncias. 


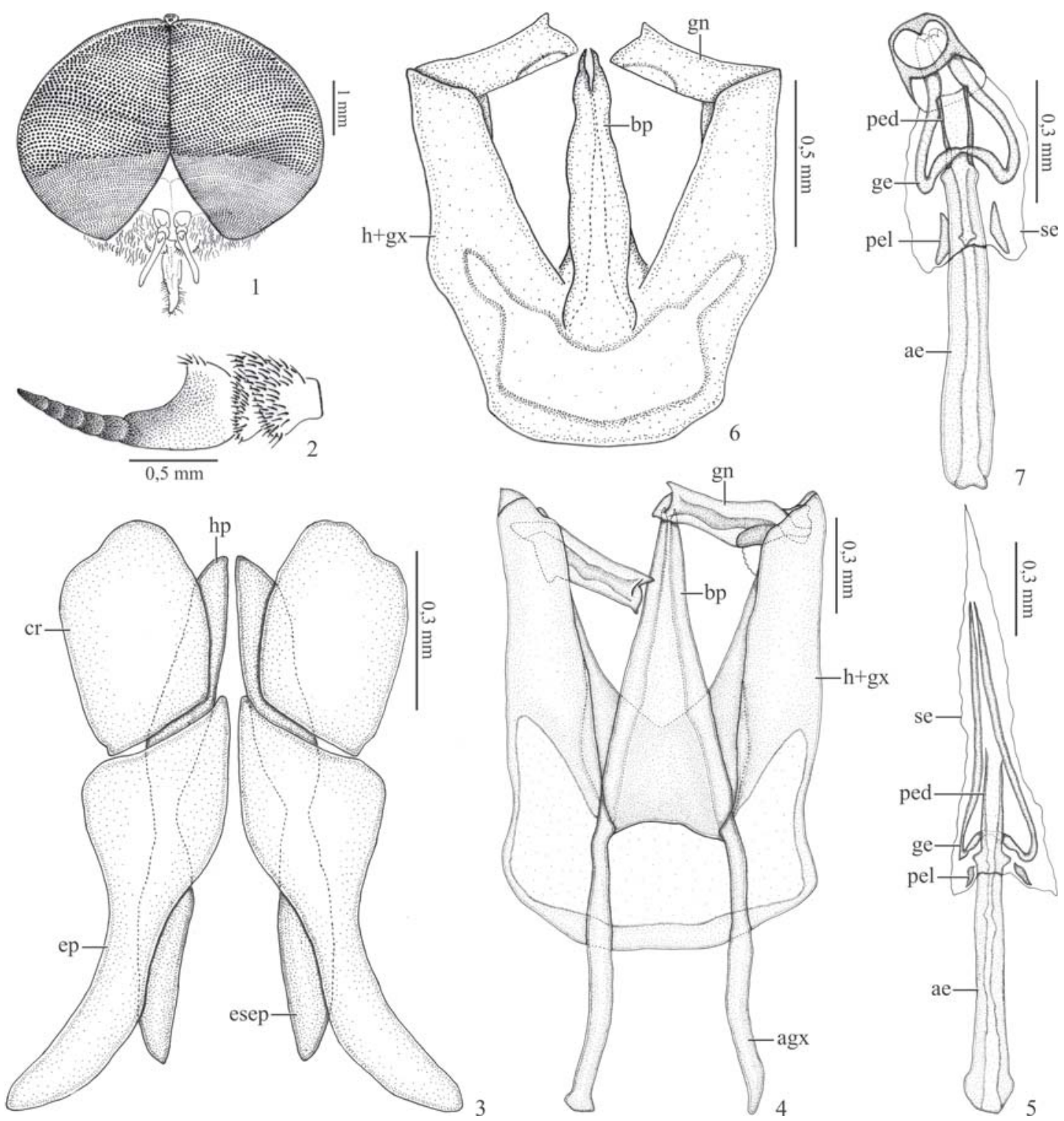

Figs. 1-7. 1-5, Leucotabanus albovarius (Walker) - Macho coletado. 1, Cabeça, vista frontal; 2, Antena, vista lateral; 3, Epândrio, cerco, hipoprocto e esclerito subepandrial, vista dorsal (cerdas não representadas); 4, Hipândrio + gonocoxitos e gonóstilos, vista dorsal (cerdas não representadas); 5, Edeago, vista dorsal. 6-7, L. albovarius (Walker) - Macho criado em laboratório (variação). 6, Hipândrio + gonocoxitos e gonóstilos, vista dorsal (cerdas não representadas); 7, Edeago, vista dorsal. (ae) apódema ejaculatório; (agx) apódema gonocoxal; (bp) bainha parameral; (cr) cerco; (ep) epândrio; (esep) esclerito subepandrial; (ge) guia edeagal (= aedeagal tine sensu Sinclair et al. 1994); (gn) gonóstilo; (hp) hipoprocto; (h+gx) hipândrio + gonocoxitos; (ped) processo endoedeagal; (pel) processo ejaculatório lateral; (se) saco espermático.

Sutura frontal esclerotinizada, com $0,77 \mathrm{~mm}$ de comprimento, sinuosa em vista ventral e pouco visível em vista lateral. Um par de cerdas orbitais anteriores, um par de cerdas orbitais posteriores, um par de cerdas verticais longas, saindo de um pequeno tubérculo e dois pares de cerdas orbitais laterais, menores. Protórax com protuberância espiracular dorsolateral de $0,67 \mathrm{~mm}$ de comprimento, ultrapassando a sutura cefalotorácica; espiráculo posicionado internamente à protuberância, semicircular, sua abertura como na Fig. 13. Mesotórax com dois pares de cerdas mesonotais, o par anterior em posição mais central e um par basal de cerdas alares. Metatórax com três pares de cerdas, duas dorsolaterais e uma lateral. Abdômen. Segmento I com espiráculo protuberante, maior que os posteriores, com dois pares de cerdas tergais e três pares de cerdas pleurais. Segmentos II-VII circundados por uma franja bisseriada de espinhos no $1 / 3$ posterior do segmento, com a série anterior, às vezes interrompida, formada por espinhos curtos e a série posterior completa, formada por espinhos mais longos e mais fortes. Espiráculos II-VII protuberantes. Segmento VIII (anal) com franjas de espinhos formando grupos distintos, como o pente pré-anal dorsolateral, com dois espinhos, um deles maior e mais desenvolvido; pente pré-anal lateral com quatro espinhos e pente pré-anal ventrolateral com cinco ou seis espinhos. Áster com um par 

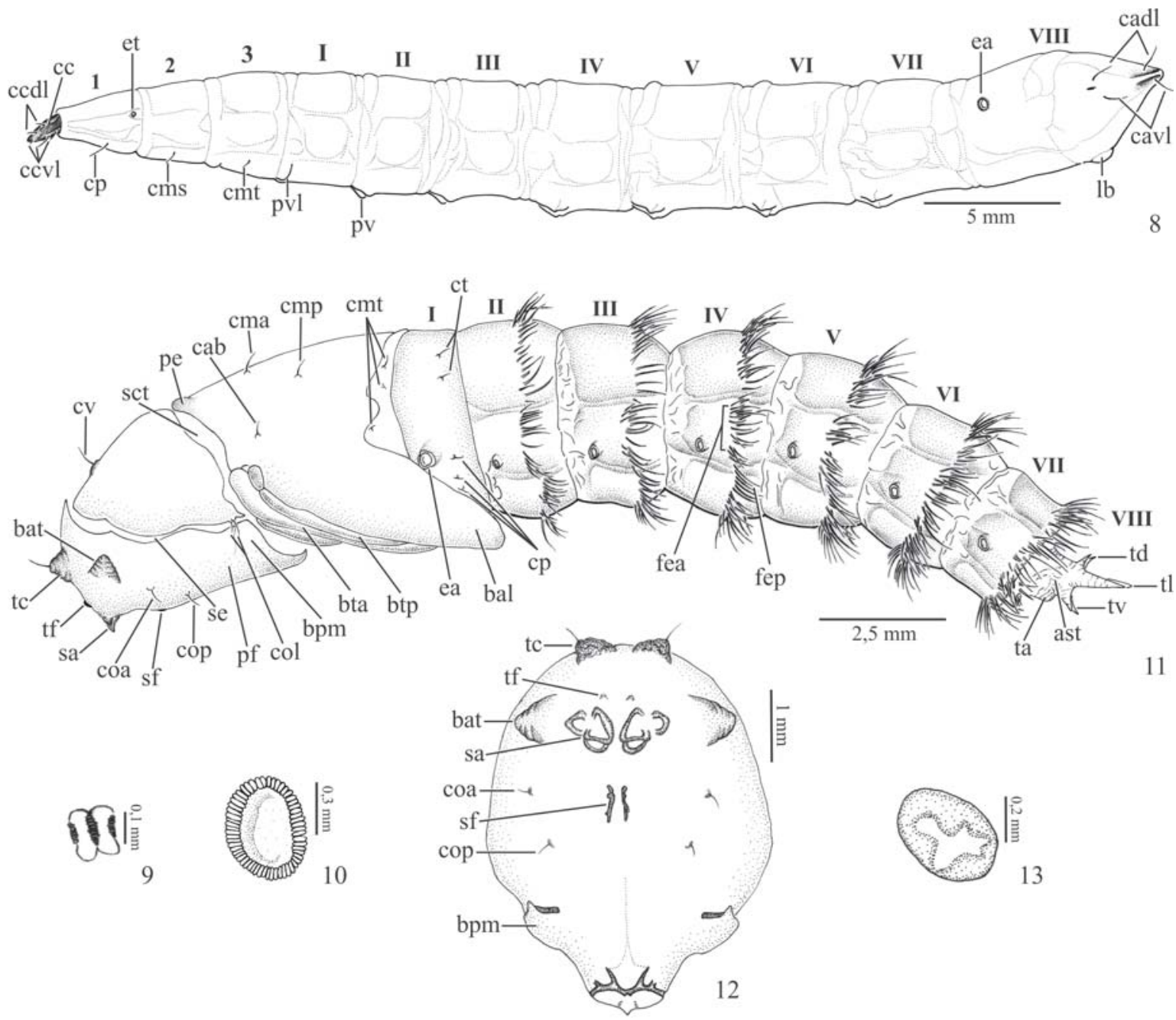

Figs. 8-13. 8-10, Leucotabanus albovarius (Walker) - Larva. 8, Habitus; 9, Espiráculo torácico; 10, Espiráculo abdominal. 11-13, L. albovarius (Walker) - Exúvia pupal (pupário). 11, Habitus; 12, Placa frontal, vista ventral; 13, Espiráculo torácico, vista lateral inclinada. (1-3) segmentos torácicos; (I-VIII) segmentos abdominais; (ast) áster; (bal) bainha alar; (bat) bainha antenal; (bpm) bainha do palpo maxilar; (bta) bainha tibial anterior; (btp) bainha tibial posterior; (cab) cerda alar basal; (cadl) cerdas abdominais dorsolaterais; (cavl) cerdas abdominais ventrolaterais; (cc) cápsula cefálica; (ccdl) cerdas cefálicas dorsolaterais; (ccvl) cerdas cefálicas ventrolaterais; $(\mathrm{cms})$ cerda mesotorácica; (cmt) cerda metatorácica; (cp) cerda protorácica; (cv) cerda vertical; (cma) cerda mesonotal anterior; (cmp) cerda mesonotal posterior; (cmt) cerdas metanotais; (coa) cerda orbital anterior; (col) cerdas orbitais laterais; (cop) cerda orbital posterior; (cp) cerdas pleurais; (ct) cerdas tergais; (ea) espiráculo abdominal; (et) espiráculo torácico; (fea) franja de espinhos anterior; (fep) franja de espinhos posterior; (lb) lobo anal; (pe) protuberância espiracular; (pf) placa frontal; (pv) pseudopódio ventral; (pvl) pseudopódio ventrolateral; (sa) saliência antenal; (sct) sutura cefalotorácica; (se) sutura epicranial; (sf) sutura frontal; (ta) tubérculo anal; (tc) tubérculo caloso; (td) tubérculo dorsal; (tf) tubérculo frontal; (tl) tubérculo lateral; (tv) tubérculo ventral.

de tubérculos dorsais curtos, direcionados para cima, com ápice levemente curvado para trás; um par de tubérculos laterais longos, projetados horizontalmente com ápice reto e um par de tubérculos ventrais um pouco maiores que os tubérculos dorsais, voltados para baixo, com ápice levemente curvado para trás. Tubérculo anal protuberante, bilobado.

Material Examinado. Adultos machos: BRASIL, Amazonas: Manaus, Sítio Vida Tropical, 0251'47"S-5955'55"W, J. A. Rafael, F. S. P. Godoi \& A. Ururahy-Rodrigues col., 07.viii.2004 [Larva]; 09.viii.2004 (Pupa); 19.viii.2004 (Ad.[Adulto]) [Coletado dentro de] tronco podre [de] Bactris gasipaes Kunth, Leucotabanus albovarius (Walker, 1854) $\sigma^{\pi}$ det. J. A. Rafael, 2004, $1 \sigma^{x}$; [Rodovia] AM 010, Km 50, 244'13"S 595' $32^{\circ} \mathrm{W}$, ii.2005, [Armadilha luminosa com] Luz mista/mercúrio, Xavier-Filho leg., Leucotabanus albovarius (Walker, 1854) $\sigma^{x}$ det. A. L. Henriques, 2005, $1 \sigma^{x}$; Presidente Figueiredo Est.[Estrada para] Balbina, [Rodovia] AM - 240, Km - 24, 02 00 '55"S, 5949'40"W, 27-28.vii.2005, [Armadilha luminosa com] Luz Mista/ Mercúrio, F. F. Xavier-Filho \& R. J. P. Machado col. Leucotabanus albovarius (Walker, 1854) $\sigma^{x}$ det. A. L. Henriques, 2005, $1 \sigma^{x}$; Estrada da Res.[Reserva] ZF-2, 01-02.xi.2005, Arm.[Armadilha] Luminosa Móvel, J. A. Rafael, F. Xavier Fo, R. J. P. Machado, A. A. Agudelo \& Y. K. Dantas [col.], Leucotabanus albovarius (Walker, 1854) $0^{\text {T }}$ det. J. A. Rafael, 2005, 3 ơ; Itacoatiara, Madeireira MIL, 024510S-583911W, 29-30.xi.2005, arm.[armadilha] luminosa móvel, J. A. Rafael, R. J. P. Machado \& A. Silva Fo, [coletado entre]19[: 00] - 20[: 00] hs, Leucotabanus albovarius (Walker, 1854) $0^{x}$ det. F. S. P. Godoi, 2005, $40^{x}$. 

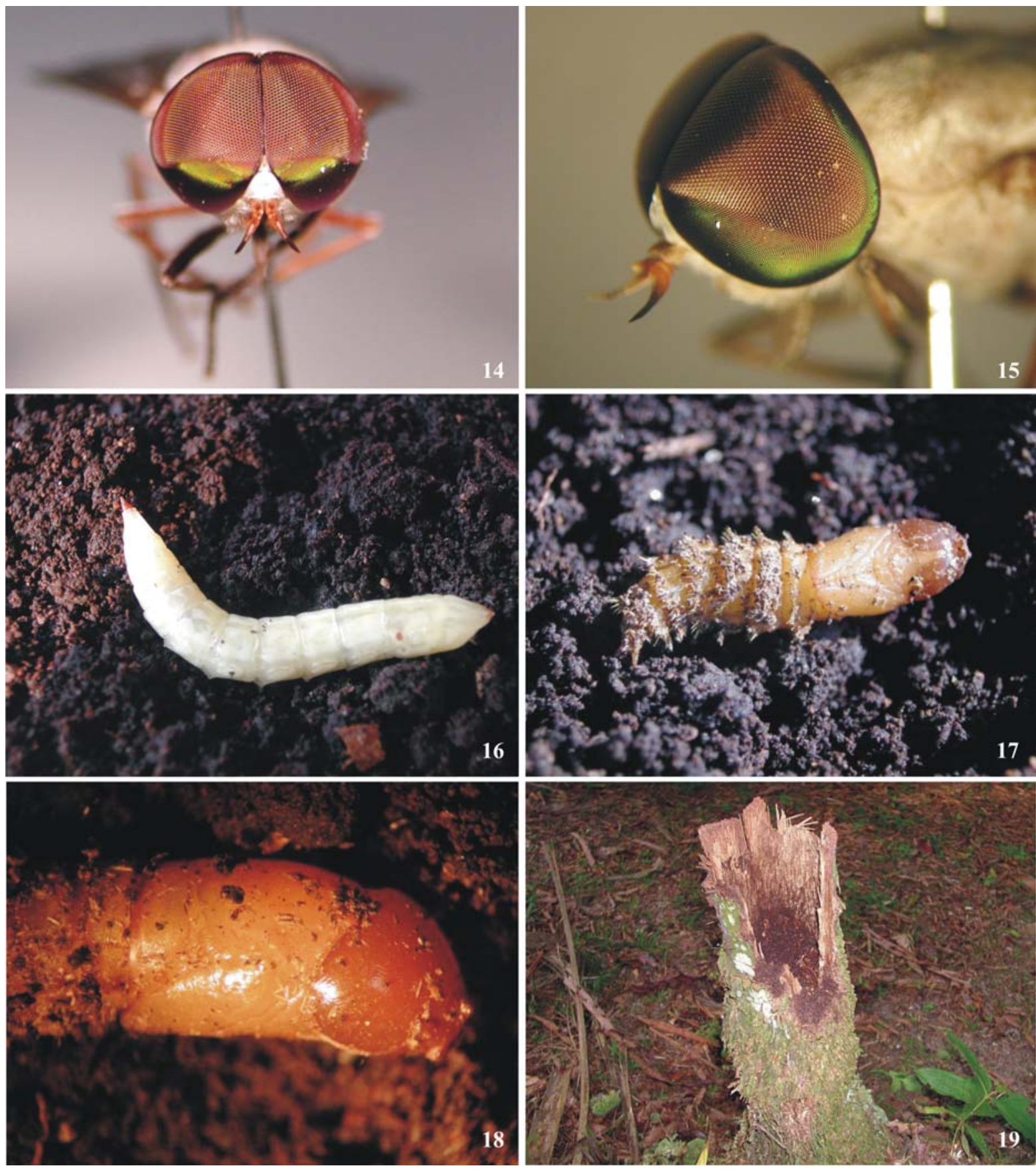

Figs. 14-19. 14-18, Leucotabanus albovarius (Walker). 14, Cabeça evidenciando coloração dos olhos, vista frontal; 15, Cabeça evidenciando coloração dos olhos, vista lateral; 16, Larva em vida; 17-18, Pupa em vida. 19, Tronco podre e aberto da palmeira Bactris gasipaes Kunth, expondo em seu interior o "paú".

Pupário: BRASIL, Amazonas: Manaus Sítio Vida Tropical, 0251'47"S-5955'55"W, 07.viii.2004 - Larva, 09.viii.2004 - Pupa, 19.viii.2004 - Adulto [Coletado dentro de] tronco podre [de] Bactris gasipaes Kunth, J. A. Rafael, F. S. P. Godoi \& A. Ururahy-Rodrigues col., Leucotabanus albovarius (Walker, 1854) $0^{x}$, det. J. A. Rafael, 2004.

Larva: BRASIL, Amazonas: Manaus, Sítio Vida Tropical, 02 $51^{\prime} 47^{\prime \prime S}-59^{\circ} 55^{\prime} 55^{\prime \prime} \mathrm{W}, 07 . v i i i .2004$ [Coletado dentro de] tronco podre [de] Bactris gasipaes Kunth, J. A. Rafael, F. S. P. Godoi \& A. Ururahy-Rodrigues col., Leucotabanus albovarius (Walker, 1854), det. J. A. Rafael, 2004
Bionomia. Duas larvas foram coletadas em um sítio localizado em área de floresta tropical úmida de terra firme pouco perturbada e pequenas áreas modificadas com plantações de árvores frutíferas, pastagem e lagos artificiais. As larvas foram encontradas em dois troncos podres de Bactris gasipaes Kunth, uma palmeira da família Arecaceae conhecida popularmente por pupunheira, que atinge cerca de 18 metros de comprimento e 25 centímetros de espessura (Ribeiro et al. 1999). Os troncos podres estavam em pé, foram derrubados e 
Tabela I. Espécies de Leucotabanus (Walker) com sexos e estágios de desenvolvimento conhecidos.

\begin{tabular}{|c|c|c|c|c|}
\hline \multirow{2}{*}{ Espécie } & \multicolumn{2}{|c|}{ Sexo } & \multicolumn{2}{|c|}{ Estágio } \\
\hline & Macho & Fêmea & Larva & Pupa \\
\hline L. albibasis (Brèthes) & $\mathrm{x}$ & $\mathrm{x}$ & - & - \\
\hline L. albovarius (Walker) & $\mathrm{x}$ & $\mathrm{x}$ & $\mathrm{x}$ & $\mathrm{x}$ \\
\hline L. ambiguus Stone* & $\mathrm{x}$ & $\mathrm{x}$ & $\mathrm{x}$ & $\mathrm{x}$ \\
\hline L. annulatus (Say)* & $\mathrm{x}$ & $\mathrm{x}$ & $\mathrm{x}$ & $\mathrm{x}$ \\
\hline L. aurarius Fairchild & - & $\mathrm{x}$ & - & - \\
\hline L. canithorax Fairchild & - & $\mathrm{x}$ & - & - \\
\hline L. cornelianus Fairchild & - & $\mathrm{x}$ & - & - \\
\hline L. exaestuans (Linnaeus) & $\mathrm{x}$ & $\mathrm{x}$ & $\mathrm{x}$ & $\mathrm{x}$ \\
\hline L. flavinotum (Kröber) & $\mathrm{x}$ & $\mathrm{x}$ & $\mathrm{x}$ & $\mathrm{x}$ \\
\hline L. itzarum (Bequaert) & $\mathrm{x}$ & $\mathrm{x}$ & - & - \\
\hline L. janinae Fairchild & - & $\mathrm{x}$ & - & - \\
\hline L. leucogaster Fairchild & - & $\mathrm{x}$ & - & - \\
\hline L. nigriventris Kröber & - & $\mathrm{x}$ & - & - \\
\hline L. pauculus Fairchild & - & $\mathrm{x}$ & - & - \\
\hline L. procallosus (Lutz) & $\mathrm{x}$ & $\mathrm{x}$ & - & - \\
\hline L. sebastianus Fairchild & - & $\mathrm{x}$ & - & - \\
\hline L. weyrauchi Fairchild & - & $\mathrm{x}$ & - & - \\
\hline
\end{tabular}

* espécies neárticas

abertos expondo a matéria orgânica em decomposição em seu interior na forma de um húmus, conhecido regionalmente como "paú", que forma um microhabitat ideal para vários organismos que o utilizam como local de alimentação e/ou reprodução ou simplesmente como abrigo (Fig. 19).

As larvas foram coletadas juntamente com grande quantidade do substrato e levadas para o laboratório onde foram acondicionadas separadamente em recipientes plásticos com o substrato coletado. Dois dias após a coleta, uma das larvas empupou e o período de pupa em condições de laboratório (média de $25^{\circ} \mathrm{C}$ ) foi de 10 dias. Logo após a emergência o olho do adulto foi fotografado e a coloração registrada antes da fixação.

Diagnose diferencial. As larvas neotropicais de Leucotabanus conhecidas, L. exaestuans (Linnaeus, 1758) e L. flavinotum (Kröber, 1934) segundo Goodwin \& Murdoch (1974) possuem comprimento entre 19-21 mm, cápsula cefálica retraída no tórax e suas estruturas, quando a cápsula cefálica é exposta, como labro, clípeo, escova cefálica, palpos maxilares, pré-mento e palpos labiais visíveis sem dissecção; apresentam ainda pubescência sobre a cutícula, meso e metatórax subiguais, sem espiráculo torácico. Abdômen com quatro pares de pseudopódios, segmento anal (VIII) menor que os segmentos abdominais, um pouco mais largo que longo, com porção posterior semi-esférica, sem espiráculo, mas provido de um sifão respiratório posterodorsal curto.

A larva de L. albovarius difere dessas espécies pelo tamanho maior (42 $\mathrm{mm}$ de comprimento), cápsula cefálica protraída, com estruturas indistintas sem dissecção, cutícula glabra, protórax mais longo que demais segmentos torácicos, com espiráculo torácico séssil, mesotórax ligeiramente menor que metatórax, abdômen com dois pares de pseudopódios, segmento anal (VIII) maior que os demais segmentos abdominais, com um espiráculo abdominal séssil, porção posterior acuminada, desprovido de sifão respiratório com cerdas anais.

Os pupários de L. exaestuans e L. flavinotum, segundo Goodwin \& Murdoch (1974), variam de 13-17 mm e 11-16 mm respectivamente, saliência antenal com uma protuberância, espiráculo torácico com abertura em forma de "C", pentes préanais dorsolateral, lateral e ventrolateral compostos por 3-5 espinhos, 6-8 espinhos e 6-8 espinhos, respectivamente e áster com tubérculo dorsal bastante reduzido.

O pupário de L. albovarius é maior, com $38,3 \mathrm{~mm}$ de comprimento, saliência antenal com 2 protuberâncias, espiráculo com abertura com forma irregular, pentes pré-anais dorsolaterais, lateral e ventrolateral compostos por 2 espinhos de tamanhos diferentes, 4 espinhos e 5-6 espinhos, áster com tubérculo dorsal desenvolvido.

Agradecimentos. A Bióloga Juliana de Souza Araújo pela revisão do manuscrito. Ao Conselho Nacional de Desenvolvimento Científico e Tecnológico - CNPq (Processo 130670/2004-9) pela bolsa de mestrado concedida ao primeiro autor.

\section{REFERÊNCIAS}

Coscarón, S.; L. C. Coscarón-Arias \& O. A. Mancebo. 1996. The immature stages of Myiotabanus barretoi Fairchild (TabanidaeDiptera-Insecta). Memórias do Instituto Oswaldo Cruz 91 21-26.

Fairchild, G. B. 1985. Notes on neotropical Tabanidae (Diptera). XVIII. The genus Leucotabanus Lutz. Myia 3: 299-331.

Fairchild, G. B. 1986. The Tabanidae of Panama. Contributions of the American Entomological Institute 22: 1-139.

Fairchild, G. B. \& J. F. Burger. 1994. A catalog of the Tabanidae (Diptera) of the Americas South of the United States. Memoirs of the American Entomological Institute 55: 1-249.

Goodwin, T. J. \& W. P. Murdoch. 1974. A study of some immature neotropical Tabanidae (Diptera). Annals of the Entomological Society of America 67: 85-133.

McAlpine, J. F. 1981. Morphology and terminology-adults, Chapter 2, p. 9-63. In: McAlpine, J. F.; B. V. Peterson; G. E. Shewell; H. J. Teskey; J. R. Vockeroth \& D. M. Wood. (eds.). Manual of Neartic Diptera. Volume 1. Monograph No. 27. Research Branch, Agriculture Canada, Otawa. (4), i-vi; 674 p.

Ribeiro, J. E. L. S.; M. J. G. Hopkins; A. Vicentini; C. A. Sothers; M. A. S. Costa; J. M. Brito; M. A. D. Souza; L. H. P. Martins; L. G. Lohmann; P. A. C. L. Assunção; E. C. Pereira; C. F. Silva; M. R. Mesquita \& L. C. Procópio. 1999. Flora da Reserva Ducke Guia de identificação das plantas vasculares de uma floresta de terra-firme na Amazônia Central. Manaus, INPA. 816 p.

Sinclair, B. J.; J. M. Cumming \& D. M. Wood. 1994. Homology and phylogenetic implications of male genitalia in Diptera - lower Brachycera. Entomologica Scandinavica 24: 407-432. 